\title{
émulations
}

\section{Marc Perrenoud (dir.) - Les mondes pluriels de Howard S. Becker}

\section{Elsa Forner-Ordioni}

Émulations - Revue de sciences sociales

2017, «Comptes rendus critiques, En ligne»

\section{Article disponible à l'adresse suivante}

https://ojs.uclouvain.be/index.php/emulations/article/view/7293

\section{Pour citer cet article}

Elsa Forner-Ordioni, « Marc Perrenoud (dir.) — Les mondes pluriels de Howard S. Becker », Émulations, en ligne. Mise en ligne le 11 juillet 2017.

DOI : 10.14428/emulations.cr.030

Distribution électronique : Université catholique de Louvain (Belgique) : ojs.uclouvain.be

(C) Cet article est mis à disposition selon les termes de la Licence Creative Commons Attribution, Pas d'Utilisation Commerciale 4.0 International. http://creativecommons.org/licenses/by-nc/4.0/

Éditeur : Émulations - Revue de sciences sociales / Presses universitaires de Louvain https://ojs.uclouvain.be/index.php/emulations

ISSN électronique : 1784-5734

UCL PRESSES

UNIVERSITAIRES 


\section{Marc Perrenoud (dir.) - Les mondes plu- riels de Howard S. Becker}

Elsa Forner-Ordioni ${ }^{1}$

Recensé : Marc Perrenoud (dir.), Les mondes pluriels de Howard S. Becker, Paris, La Découverte («Recherches »), 2013, 237 p.

Paru en 2013, Les mondes pluriels de Howard S. Becker salue l'œuvre du chef de file de la deuxième école de Chicago à l'aube de son $90^{\mathrm{e}}$ anniversaire. Inspiré d'un colloque qui s'est tenu à l'université de Lausanne en octobre 2011 sur l'héritage interactionniste en sociologie du travail, l'ouvrage collectif invite, d'une part, à explorer de nouveaux terrains ethnographiques de la sociologie des professions sous l'angle de la tradition interactionniste (chapitres 1 à 7) et d'autre part à réfléchir au contexte d'émergence et à la place des travaux d'Howard Becker dans le champ de la recherche en sciences sociales (chapitres 8 à 13). Marc Perrenoud propose une « exploration approfondie » de la pensée interactionniste (p. 8) qui semble particulièrement d'actualité, alors que vient de paraître un autre ouvrage aux problématiques voisines, Andrew Abbott et l'héritage de l'école de Chicago (Demazière et Jouvenet, 2016). Les apports majeurs des travaux d'Howard Becker sont ainsi mis à l'épreuve sous l'angle de la méthode, des concepts et, en tout premier lieu, de l'enquête de terrain et de la sociologie des professions.

Nous verrons d'abord comment cet ouvrage travaille, terrains à l'appui, l'héritage de la pensée interactionniste avant de détailler la portée heuristique de la démarche et d'en discuter les apports et les critiques.

\section{L'apport de la sociologie interactionniste : un art subtil du déplacement.}

L'ouvrage nous propose un panaché des terrains actuels inspirés de la sociologie interactionniste. La machine n'est pas grippée : la grille interactionniste, avec sagacité, se révèle toujours aussi efficace pour analyser, par exemple dans le chapitre 1, le phénomène du dopage chez les cyclistes professionnels. La première partie de l'ouvrage est ainsi consacrée au savoir-faire délicat de l'enquête ethnographique telle que la conçoit Becker : faire de la sociologie, c'est d'abord «aller à la rencontre d'objets originaux » (p. 5).

${ }^{1}$ Doctorante au Centre d'étude des mouvements sociaux, École des hautes études en sciences sociales. 
C’est avec la virtuosité sensible d'un H. Becker dans «Qu'est-ce qu'on joue maintenant?» Le répertoire de jazz en action (2011) que M. Perrenoud décode dans le chapitre 5 les interactions d'un espace informel et déplie - avec une certaine poésie - la façon dont les musiciens, entre improvisation et partition, à l'image du standard de jazz, évoluent dans des espaces construits par les interactions qui sont au centre « là où barmen et serveuse travaillent en même temps que les musiciens à construire «ce qui se passe» (p. 89). Il y décrit très bien les résultats que l'on peut attendre d'une telle sociologie : les événements sont davantage une affaire de degré que de mondes étanches. Il y a de l'artisanat dans l'art autant qu'il y a du collectif dans la production d'une œuvre.

L'héritage interactionniste semble s'imposer avant tout comme un art savant du coqà-l'âne. Dans la sociologie de Hughes comme dans celle de Becker, l'exploration de la diversité du monde social apparaît à travers « l'emploi systématique de la comparaison et des rapprochements les plus inattendus» comme le souligne Pierre-Henri Menger (p. 207). Cyclistes (chap. 1) et policiers (chap. 9) côtoient médecins, (chap. 3) tatoueurs (chap. 4) et professionnels des ressources humaines (chap. 2), alors qu'un groupe de musiciens qui joue pour une soirée du Rotary Club se délecte d'un coq au vin derrière un paravent entre deux standards de jazz (chap. 5). Sur la table à dissection des interactionnistes, il y a quelque chose de « la rencontre fortuite entre une machine à coudre et un parapluie », à l’image de la poésie surréaliste selon Lautréamont. Cet inventaire de métiers nous rappelle au bon souvenir d'un autre père de l'École de Chicago, lui-même indissociable de l'œuvre de Howard Becker. Everett Hughes plane en effet au-dessus de l'ouvrage, en maître incontesté du genre : «Étudier les métiers en les comparant permet de mieux comprendre le travail des médecins en étudiant celui des plombiers, celui des prostituées en étudiant celui des psychiatres » (Hughes 1996 : p. 87). À partir d'une démarche ancrée dans une dimension comparative, les professions se réfléchissent en miroir, comme par concaténation : un métier en appelant un autre.

La trame de l'ouvrage respecte ainsi la richesse des terrains sans vouloir administrer la preuve par le concept. Plutôt que de céder à la tentation du dévoilement, c'est par le décalage, en multipliant les regards qu'on saisit le mieux la variété d'une situation. Cette première partie nous donne donc une vue d'ensemble kaléidoscopique, où la réalité gagne à être perçue comme « une multiplication de perspectives et de significations » (p. 212), tout en conservant une inclinaison pour le casse-tête où la sociologie au plus près du terrain se mue en dilemme métaphysique, pour ces patients qui attendent un médecin : "piégés par la contradiction interne entre d'un côté, leur irritation pour la perte de temps (...) et de l'autre, le sentiment que le retard du médecin atteste du temps qu'il consacre au patient », car «ce qui est exceptionnel pour l'un est une routine pour l'autre» (p. 58). À l'image d'un ruban de Moebius dont la perspective mouvante redonne une place centrale à ce qui semblait anecdotique, l'œil sociologique des interactionnistes nous permet de saisir ce dont la sociologie est capable pour rendre compte des situations et des interactions dans lesquelles les individus sont pris. 


\section{« Doing things together » : la portée heuristique de la méthode interactionniste.}

On comprend ainsi au fil de la lecture à quel point la sociologie interactionniste et la sociologie des professions entretiennent des liens ténus : tel un tableau impressionniste fait de «touches successives » (p. 49), chaque article s'attèle à restituer finement ce que signifie pour chacun et pour tous le fameux adage «doing things together». Les coulisses de chaque métier révèlent à leur tour une fenêtre heuristique sur un monde social qu'il nous est donné de découvrir, non de façon laborieuse, mais comme en regardant par le trou de la serrure - le lecteur ainsi guidé par un voyeurisme bienveillant qui fait la force de la sociologie de Becker : la curiosité de connaître d'autres mondes.

Reste à savoir comment décrire ces «choses que les gens font ensemble»: l'autre force du travail de Becker est justement, comme le montre la deuxième partie, de conserver une souplesse dans le maniement de concepts et une méfiance à l'égard des généralisations rigides et des résultats figés. Dans le monde que Becker s'attache à décrire, le réel ne se prête pas à l'élaboration de théories totalisantes : «le monde est plus complexe qu'on ne le croit » (p. 202) : l'essentiel est dans le détail.

Sous cet angle, la deuxième partie du livre discute plus particulièrement l'approche de Becker, dont le savoir-faire serait le «travail d'un homme de métier bien plus que celui d'un professionnel de la sociologie » (p. 200). La conception que Becker a du travail sociologique est incarnée dans une forme d'artisanat qui oscille entre souci de transmission et modelage des concepts à la pièce. La question de la transmission tient en outre une place importante dans l'ouvrage qui a le mérite de rassembler des chercheurs qui ont contribué à la popularisation de ses travaux en France, tels qu'Anne-Marie Arborio (chapitre 8) et Jean-Michel Chapoulie (chapitre 11). Ces deux chapitres offrent un large panorama historique de l'émergence des concepts d'Howard Becker dans le contexte français.

H. Becker, lui-même auteur d'un chapitre, décrit «l'esprit » de l'interactionnisme, où il s'agit d'étudier les disputes, car «le sociologue n'en apprend jamais autant que lorsque les participants sont en désaccord» (p.120). Il invite à suivre les objets par les endroits où ils passent, à travailler sur les controverses pour comprendre en creux ce à quoi tiennent les individus. Les pistes qu'il dessine font résonner les apports récents de la sociologie des sciences, des valuation studies et de la sociologie pragmatique, à la croisée de l'interactionnisme symbolique et des pragmatistes anglo-saxons. Il s'agit là de dépasser les «rhétoriques professionnelles» au profit d'une compréhension de la formation des valeurs, des processus historiques, des modes d'attachement et d'engagement ou encore du «vrai boulot» (Gomart et Hennion, 1998; Bidet, 2011; Dewey, 2011) pour comprendre, à rebours, comment «la force du sentiment d'appartenance au groupe professionnel peut se mesurer à la capacité de partager la sensibilité à l'égard du risque d'erreur» (p. 231). 


\section{L'angle mort de la critique : sociologie interactionniste et rapports sociaux.}

La richesse et la diversité de l'opus laissent pourtant en suspens des questionnements plus anciens : quel est le projet critique de la sociologie interactionniste ? comment éviter l'écueil de «l'effet catalogue» d'enquêtes qui n'existeraient que par et pour ellesmêmes, peinant à envisager un projet réflexif de plus grande envergure? (Crozier et Friedberg, 1977)?

La deuxième partie de l'ouvrage s'attache finalement à dessiner dans la description des relations entre concept, méthode et terrains d'enquête une sociologie à l'image des métiers dont elle est férue, façonnée au cas par cas, privilégiant toujours la spécificité à la généralisation. Cette modestie épistémologique, au plus près de la description plutôt que de la recherche des causes, privilégiant le «comment» au «pourquoi » avoisine la théorie ancrée de Glaser et Strauss. C'est donc après un hommage fouillé et riche, à l'endroit où on l'attend, que la dimension critique de l'ouvrage intervient et prend ses distances avec une sociologie des professions qui sépare "petits métiers » et "professions prudentielles» (Champy, 2009), se risquant ainsi à figer ce à quoi Hughes avait justement tenté d'échapper, arguant « [qu’] il faut nous débarrasser de toutes les notions qui nous empêchent de voir que les problèmes fondamentaux que les hommes rencontrent dans leur travail sont les mêmes, qu'ils travaillent dans un laboratoire illustre ou dans les cuves malpropres d'une conserverie» (Hughes 1996: 80). Pour autant, défendre que les problèmes rencontrés sont les mêmes dans la plupart des métiers permet-il de rendre compte dans le même temps des effets de prestige des métiers dans l'espace social ? Par là même, la capacité singulière de cette sociologie à saisir de l'infiniment petit suffit-elle à restituer le sens du travail pour les individus ? En a-t-elle jamais eu l'ambition? Tout se passe comme si le pari de la sociologie interactionniste visait à décrire les métiers depuis les marges, à la manière d'Andrew Abbott qui invite à partir des «frontières » pour laisser au lecteur le soin d'en comprendre le cœur.

Le chapitre 6, écrit par Marie Buscatto, s'attache à démontrer comment la sociologie interactionniste peut intégrer de nouveaux questionnements et travailler la question des inégalités sociales sans pour autant renoncer à la description de l'activité en train de se faire. Au milieu d'enquêtes qui témoignent de la vivacité de l'approche interactionniste par la diversité des métiers étudiés, ce chapitre laisse entrevoir un espoir - à mi-parcours - quant à la capacité de la sociologie interactionniste à interroger de manière réflexive la question des inégalités. Auteure d'une enquête sur les femmes dans le jazz, Marie Buscatto a la tâche de convaincre que le concept de « monde de l'art », par sa plasticité, se serait ainsi « enrichi de nouvelles problématiques peu présentes dans la réflexion initiale de Becker posée au début des années $80 »$ (p. 112). Force est de constater que malgré cette incursion brève dans le monde des musiciennes de jazz, les mondes pluriels d'Howard Becker se déclinent, à l’instar des tatoueurs, musiciens, cyclistes, chirurgiens, DRH et policiers, exclusivement au masculin, exception faite de 
l'enquête sur les aides-soignantes d'Arborio, prise comme exemple de cheminement méthodologique davantage que comme objet heuristique. En rendant invisibles les rapports sociaux au profit d'une attention particulière aux interactions et aux situations, la sociologie interactionniste, dans toute sa capacité virtuose à décrire la variété des mondes sociaux et à en retranscrire par achoppement sensible les ficelles, semble contribuer à son tour à produire un monde qui ne serait digne d'intérêt que lorsqu'il est construit par des hommes (Monjaret et Pugeault, 2014; Copans, 1999). Coïncidence ou reproduction? Si Buscatto et Menger tentent de dépasser cette critique dans la deuxième partie de l'ouvrage, la contradiction est d'autant plus forte que la thématique demeure absente des différentes enquêtes - et l'on sait que Becker tient en estime l'empirie bien plus que la théorie. La réalité décrite semble ainsi continuer de contourner la dimension agonistique inhérente aux mondes professionnels dont il est question. "Comment loger la condition des différences et des inégalités de condition sociale dans un cadre essentiellement relationnel? La réponse donnée par Hughes est entièrement dépendante de son objet d'analyse : le travail.» (p. 208). Pour autant, « les “

réalités épaisses" que sont les institutions ne sont pas dotées d’un pouvoir de détermination des comportements et des individus », note Pierre-Michel Menger dans le dernier chapitre (p. 209). La sociologie interactionniste est-elle encline à produire des enquêtes sans prendre en charge le travail de réflexivité nécessaire au choix des objets abordés? Le tableau dressé semble d'autant plus monochrome que dans les métiers choisis et étudiés au sein de l'ouvrage, les dimensions du genre, de la classe et de la race, non exploitées, apparaissent en filigrane comme si elles ne méritaient pas qu'on s'y intéresse, ni du point de vue du processus (comment un milieu professionnel devientil ou demeure-t-il non-racisé ou masculin ?) ni de celui des effets (que fait le genre aux sociabilités professionnelles ?).

\section{Bibliographie}

ARBORIO A-M. (2012), Un personnel invisible. Les aides-soignantes à l'hôpital, Paris, Economica («Sociologiques »).

BECKER H. (1985), Outsiders. Études de sociologie de la déviance, Paris, Métailié.

BECKER H. (2011), Qu'est-ce qu'on joue maintenant ? Le répertoire de jazz en action, Paris, La Découverte.

BIDET A. (2011), L'engagement dans le travail. Qu'est-ce que le vrai boulot ? Paris, Presses Universitaires de France.

CHAмpy F. (2009), La sociologie des professions, Paris, Presses Universitaires de France.

Copans J. (1999), L’enquête ethnologique de terrain, Paris, Nathan Université («128»).

Crozier M. et Friedberg E. (1977), L’acteur et le système, Paris, Édition du Seuil. 
Demaziere D. et JouvenEt M. (2016), Andrew Abbott et l'héritage de l'école de Chicago, Paris, Volume 1, EHESS («Temps et lieux »).

DeWEY J. (2011), La formation des valeurs, Paris, Les empêcheurs de penser en rond.

GOMART E. et Hennion A. (1999), "A sociology of attachment: Music Lovers, Drug Addicts », The Sociological Review, Volume 47, Issue S1, p. 220- 247.

HuGHES E. C. (1996), Le regard sociologique. Essais choisis, Paris, Les Éditions de l'EHESS.

Monjaret A. et Pugeault C. (2014), Le sexe de l'enquête, approches sociologiques et anthropologiques, Lyon, ENS Éditions. 